\title{
도 QN
}

Revista GEON (Gestión, Organizaciones y Negocios). ISSN: 2346-3910 en línea revistageon@unillanos.edu.co Universidad de los Llanos Colombia

Martínez, Carolina Esther; Ortiz Moreno, Gina Zoraida;

Hernández, José Williami.

Efectos en los estados financieros de la implementación de la gestión ambiental en el molino industrial arrocera superior limitada.

Revista GEON, Vol. 3, No. 2, 2016 Pág. 14-20

Disponible en: $\underline{\text { https://doi.org/10.22579/23463910.56 }}$

${ }^{\mathrm{i}}$ https://orcid.org/0000-0003-1173-5021

Esta publicación se encuentra bajo licencia: Creative Commons

ReconocimientoNoComercialSinObraDerivada 4.0 Internacional
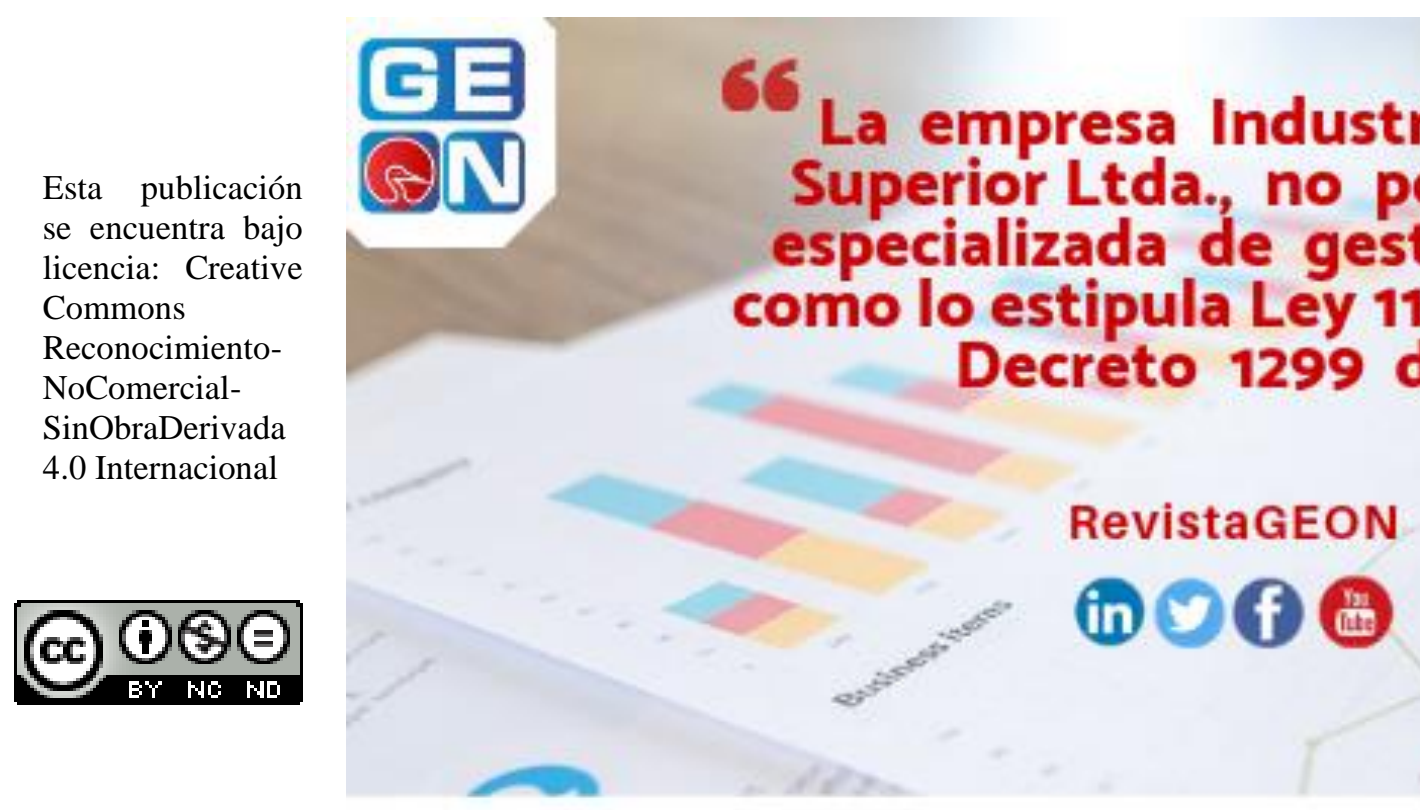


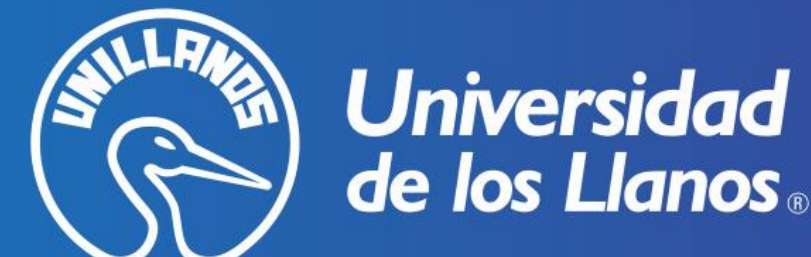

Compromiso con la paz y el desarrollo regional

\section{Revista}

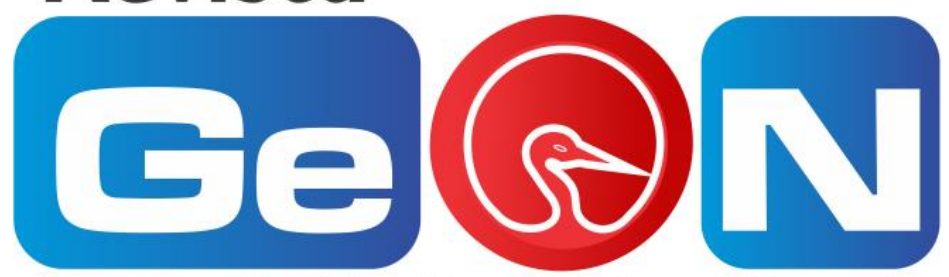

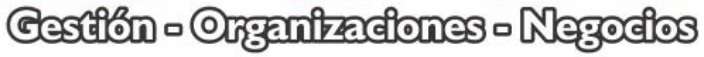

\section{ISSN 2346-3210 \\ Volumen 3No2 Julio-Diciembre2016}

Revista Electrónica de la Facultad de Ciencias Económicas de la Universidad de los Llanos VILLAVICENCIO - COLOMBIA

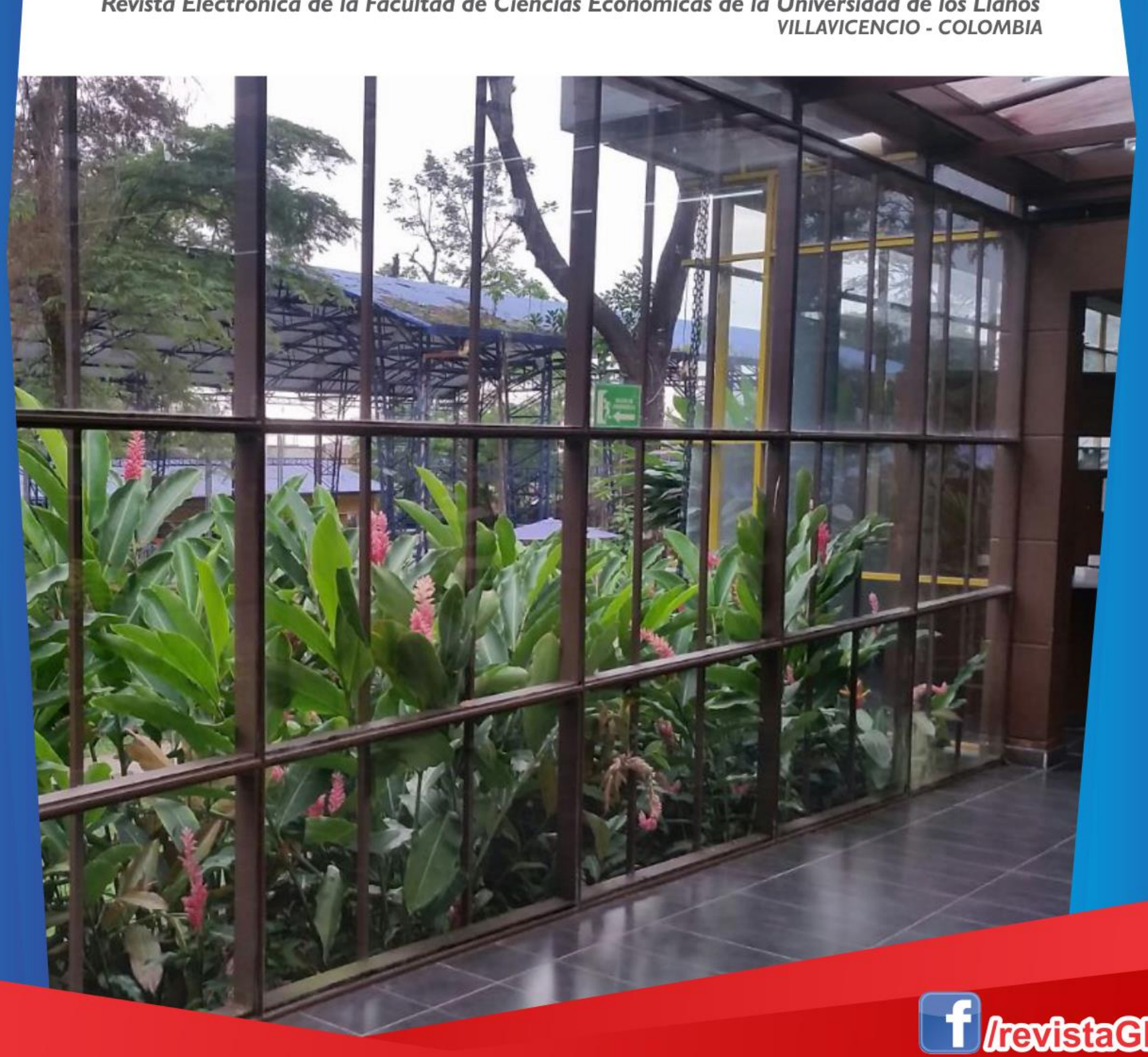

$\stackrel{\frac{m}{7}}{\frac{m}{7}}$

Qய

像

.




\section{Efectos en los estados financieros de la implementación de la gestión ambiental en el molino industrial arrocera superior limitada por Carolina Martínez - Gina Ortiz - José Hernández}

Carolina Esther Martínez

Contador Público (e)

Universidad Cooperativa de Colombia

Carolinae.martinez@campusucc.edu.co

Gina Zoraida Ortiz Moreno

Contador Público (e)

Universidad Cooperativa de Colombia

ginazortiz@gmail.com

José William Hernández González

Contador Público

Docente Universidad de los Llanos

jwhernandez@unillanos.edu.co

\section{Resumen}

El Molino Industria Arrocera Superior LTDA, no tiene creado el Departamento de Gestión Ambiental DGA para dirigir la gestión ambiental como lo exige la Ley 1124 de 2007 y el Decreto 1299 de 2008, que permita velar por el cumplimiento de la normatividad ambiental; prevenir, minimizar y controlar la generación de cargas contaminantes hacia el entorno. Esta problemática le genera a la empresa, una serie de situaciones adversas que afecta su desarrollo organizacional y además puede acarrearle posibles sanciones por el incumplimiento de las normas. Por lo anterior, el estudio tiene como objetivo, determinar los efectos en los estados financieros que podrían darse por la implementación de la gestión ambiental en la empresa. Se empleó la investigación descriptiva, con la cual se reseñó la información recolectada a través de encuestas, libros y documentos relacionados con el tema. Resultados. El 80\% de los encuestados, dicen que existe incumplimiento en leyes ambientales, como la que exige la creación del DGA y la implementación del sistema de gestión ambiental; y un $60 \%$ dice que esto se debe a la falta de capacitación del personal y al desarrollo de una gestión que permita mitigar los efectos negativos hacia el medio ambiente, por las actividades. Se concluyó que la empresa no realiza un proceso de gestión ambiental mediante un departamento especializado como lo exige la ley; y que, al proyectar su implementación, se concluyó que trae efectos para el crecimiento empresarial.

Palabras clave. Gestión ambiental, departamento de gestión ambiental, efectos, prevención, implementación, minimización, normatividad, ambiente.

\begin{abstract}
Superior Rice Mill Industry LTDA, has created the Department of Environmental Management DGA to direct environmental management as required by the 1124 Act of 2007 and Decree 1299 of 2008, which allows ensuring compliance with environmental regulations; prevent, minimize and control the generation of pollutant loads to the environment. This issue generates the company to a number of adverse situations that affect their organizational development and can also acarrearle possible sanctions for non-compliance. Therefore, the study aims to determine the effects on the financial statements could be taken for the implementation of environmental management in the company. Methodology. Descriptive research was used, with which the data collected through surveys, books and documents related to the subject was reviewed. Results. $80 \%$ of respondents say there is default in environmental laws, as required by the creation of the DGA and the implementation of environmental management system; and $60 \%$ say that this is due to lack of staff training and the development of governance to mitigate the negative effects for the environment, for the activities. Conclusions. The company does not perform a process of environmental management through a specialized department as required by law; and that the project implementation, it was concluded that brings effects for business growth.
\end{abstract}


Efectos en los estados financieros de la implementación de la gestión ambiental en el molino industrial arrocera superior limitada por Carolina Martínez - Gina Ortiz - José Hernández

Keywords: Environmental management, environmental management department, effects, prevention, implementation, minimization, regulations, environment

\section{Introducción}

La preocupación que durante años se ha generado por la conservación y protección del medio ambiente, llevó a que, en el año 2007, el gobierno central diseñará y sancionará la ley 1124, la cual obliga a las empresas del nivel industrial la creación de un Departamento de Gestión Ambiental (DGA), área que se encarga de velar por el cumplimiento de la normatividad medioambiental de la República. Aunque dicha ley fue objeto de múltiples demandas, la Corte Constitucional, en su sentencia C-486 del año 2009, dejó en firme su aplicación, declarando únicamente como inexequible la expresión "todas" contenida en el artículo 8. Sin embargo, la norma no segmenta a las industrias por sus niveles de contaminación ni de toxicidad, sino por su tamaño en cuanto a cantidad de empleados y activos, sin tener en cuenta sus niveles de solvencia económica o su capacidad presupuestal para asumir un proceso tan estricto y que podría impactar, ya sea positiva o negativamente, sus estados financieros.

La industria molinera del arroz del departamento del Meta no se encuentra exenta de la aplicación de la referida ley 1124 del 2007, por lo que el Molino Industria Arrocera Superior LTDA, ubicado en el municipio de Acacias, en el departamento del Meta, debe dar cumplimiento a dicha norma. No obstante, dicha empresa no emite una cantidad considerable de contaminación ni de desechos tóxicos peligrosos, pero ya que la legislación no tiene en cuenta estos factores, debe sujetarse a la literalidad de la ley con el fin de evitar sanciones de diferentes tipos.

El problema radica en la forma en que la gestión ambiental podría impactar la situación económica del Molino Industria Arrocera
Superior LTDA y, por ende, sus estados financieros, ya que los Departamentos de Gestión Ambiental requieren de una inversión para su creación y manutención, lo que le generaría mayores costos y gastos a la empresa, creando la posibilidad de generar situaciones de insolvencia e iliquidez o disminuyendo las utilidades contables, fiscales y financieras del periodo.

Bajo esta problemática se efectuó un diagnóstico de cómo se encuentra la organización, con respecto a las normas de gestión ambiental; se realizó un análisis financiero de los estados contables de la empresa, de los dos últimos años; se planteó el proceso y las actividades generales para la implementación de la gestión ambiental en la empresa y se definió los posibles cambios en las principales cuentas contables de los estados financieros y la repercusión que tendría en el desarrollo de la empresa, si se llegase a implementar el proceso y las actividades planteadas de la gestión ambiental. Es necesario la investigación, pues permite conocer que tan benéfico resulta para este tipo de organizaciones ejecutar acciones tendientes a la preservación del medio ambiente y los recursos naturales, en cumplimiento de la ley 1124 del 2007.

\section{Desarrollo}

\section{Fundamentación Teórica}

Para el tema que atañe a la investigación, es decir la gestión ambiental en la empresa Molino Industrial Arrocera Superior LTDA, organización mediana que no cuenta con un Departamento de Gestión Ambiental por lo que la Corte Constitucional $^{2}$ establece lo siguiente:

"La Ley 1124 de 2007 que regula la profesión de administrador ambiental, en su artículo $8^{\circ}$ incluye una disposición que obliga a todas las empresas del sector industrial a crear un departamento de gestión ambiental con el objeto de velar por el cumplimiento de la normatividad sobre la materia, medida que si bien propende por 
Efectos en los estados financieros de la implementación de la gestión ambiental en el molino industrial arrocera superior limitada por Carolina Martínez - Gina Ortiz - José Hernández

un fin constitucionalmente legítimo, resulta desproporcionada respecto de ciertas empresas, particularmente las pequeñas empresas y las microempresas, que con una capacidad adquisitiva restringida, en las que los asuntos relacionados con la administración, producción y ventas son elementales y reducidos, además de un acceso limitado al sector financiero, con sistemas de fabricación prácticamente artesanales, para las que el cumplimiento de la obligación impuesta puede generarles la quiebra." (Congreso de la República, 2007).

Si bien es cierto que el Departamento de Gestión Ambiental se encarga de velar por el cumplimiento de la normatividad ambiental con el fin de minimizar afectación del medio ambiente a través de cargas contaminantes y el uso racional de los recursos deja un vacío en su intervención puesto que no incluye a las micro y pequeñas empresas industriales sin establecer la cantidad de desechos contaminantes que estas pueden generar al medio ambiente al no tener control de las mismas y esto puede afectar el desarrollo económico empresarial y su imagen corporativa. De esta manera y como indica Vásquez (2009), la Gestión Ambiental Empresarial "es un factor crucial que influye decisivamente tanto en la imagen corporativa de la empresa, como en la calidad del producto, en el costo de comercialización y, a lo sumo, la competitividad" $(\mathrm{p}, 1)$ que son factores básicos para obtener el éxito organizacional en mercados complejos y competitivos, como el arrocero en donde desarrolla sus actividades la empresa en estudio Industria Arrocera Superior LTDA, la cual requiere realizar la implementación de la gestión ambiental para establecer los posibles efectos en sus estados financieros en el año siguiente.

Para ello, es importante realizar un análisis financiero del escenario actual de la empresa, para lograr tener perspectiva de los posibles efectos que podría causar la implementación de una propuesta del proceso de gestión ambiental. Este análisis financiero incluiría el Método de Análisis Vertical o Estático que según Alarcón y Ulloa (2012). "es un análisis estático que se emplea para examinar estados financieros como el balance general y el estado de resultados de un período, para conocer su situación o resultados, comparando las cifras en forma vertical" ( $p, 10)$ para conocer la distribución de los activos y el manejo de las deudas en la empresa en los años anteriores.

También se empleó el Análisis Horizontal que "es un procedimiento que consiste en comparar estados financieros homogéneos en dos o más períodos consecutivos. En este método se utiliza la técnica de Aumento y disminuciones, para determinar los aumentos y disminuciones o variaciones de las cuentas, de un período a otro" (Alarcón y Ulloa, 2012, p 19-20), el cual es importante porque se puede determinar los cambios en las cuentas entre periodos contables.

\section{Metodología}

El tipo de investigación aplicado fue el descriptivo, que según Tamayo (2004) "trabaja sobre realidades de hechos, y su característica fundamental es la de presentar una interpretación correcta." Este proyecto se describió la forma de cómo afecta en los estados financieros, la implementación del departamento de gestión ambiental de la empresa.

Las fuentes de información utilizadas para el desarrollo de la investigación, fueron las primarias por medio de encuestas cuyos cuestionarios Se diseñó un cuestionario en donde se plasmaron las características y requisitos que establecen las normas para las empresas que deben cumplir con la gestión ambiental, para lo cual se solicitó una reunión con el gerente de la entidad para que conteste el cuestionario. Teniendo en cuenta las respuestas afirmativas dadas por el gerente en el cuestionario, se relacionó las características y requisitos legales que tiene efectivamente la empresa con los establecidos en la legislación, de esta forma, se puede determinar claramente cuáles son las normas que le atañen a la empresa.

También, se emplearon las fuentes secundarias como los libros, internet, revistas, documentos institucionales, como los Estados Financieros para establecer la situación financiera de la 
Efectos en los estados financieros de la implementación de la gestión ambiental en el molino industrial arrocera superior limitada por Carolina Martínez - Gina Ortiz - José Hernández

empresa, teniendo en cuenta como puntos de partida el balance general a corte a 31 de diciembre de 2013 y 2014.

\section{Resultados}

Los resultados de la aplicación de la encuesta indican que en: un $80 \%$ de los empleados y directivos encuestados afirman que no existe creado un Departamento de Gestión Ambiental, como lo exige la legislación ambiental y esto le puede acarrear las sanciones respectivas por esta falta de aplicación. (Ver gráfica 1).

Un $100 \%$, de los encuestados cree que al estructurarse el Departamento de Gestión Ambiental DGA se fortalece la función de protección al medio ambiente, pero que requiere de un recurso humano capacitado y especializado en el tema, porque en la actualidad sus competencias no llenan este requisito, a pesar de que a existen los recursos monetarios para avanzar en el desarrollo de la aplicación de la normatividad y la creación del área especializada, con lo cual se podrían dar posibles cambios en las principales cuentas contables de los estados financieros $\mathrm{y}$ por ende en el desarrollo $\mathrm{y}$ crecimiento de la empresa.

La Industria Arrocera Superior Ltda. con Nit 892003074-5 ubicada en el municipio de Acacias en el departamento del Meta, es una empresa fue creada el 22 de abril de 1986 en Acacias Meta, inscrita en cámara de comercio, constituida como persona jurídica constituida por dos personas; y tiene como objetivo social las siguientes actividades: compra, procesamiento, venta, distribución, exportación de arroz y/u otros cereales, explotación agrícola y/u otros cereales.

En el caso de las empresas industriales arroceras, en los últimos años el gobierno nacional, ha emitido una serie de normatividad como estrategia para fomentar la colaboración y el apoyo de los empresarios arroceros, en la reglamentación e implementación de áreas especializadas en las organizaciones para proteger el medio ambiente. Y que al evaluar la aplicabilidad de estas normas se logró establecer que existe un cumplimiento parcial del Decreto 1713 de 2002 que se refiere al desarrollo de la Gestión integral de residuos en cuanto a la recolección y almacenamiento de residuos sólidos producidos por la actividad industrial que lleva a esta organización, porque no son adecuados los recipientes, depósitos contenedores retornables en donde se almacenan los residuos mientras se procesan para su aprovechamiento, transformación, comercialización, tratamiento o disposición final; y esto genera afectos al medio ambiente como al aire por la expedición de olores, al suelo por los liquido o lixiviados, lo cual trae consecuencias para la salud de los empleados y para la población de influencia cercana a la ubicación de la organización.

Otra norma que se está incumpliendo parcialmente es la Ley 1252 de 2008, la cual dicta normas prohibitivas en materia ambiental, referente a los residuos sólidos; y tampoco se cumple con la exigencia de la ley 1124 y su Decreto Reglamentario 1299 de 2008, que obliga a las empresas del nivel industrial la creación de un DGA que es precisamente el área que se encarga de velar por el cumplimiento de la normatividad medioambiental emitida por el Estado, y que por la no existencia o la implementación en la organización de este departamento, en la actualidad todavía existe incumplimiento de legislación, lo cual puede traer efectos y posibles sanciones, si no se adelanta el proceso para la puesta en marcha del DGA.

En el análisis horizontal se determinó un aumento entre el año 2013 y 2014 de $26 \%$, lo que indica un incremento importante para para dar respaldo a las deudas contraídas. Sin embargo, el resultado del análisis en el grupo del Pasivo, arrojó un incremento del $35 \%$ lo cual indica mayor endeudamiento y un menor activo para su respaldo. Lo anterior indica que, en el año 2014, en las cuentas del activo se incrementó el valor de las cuentas por cobrar; en el pasivo las cuentas por pagar y considerablemente la cuenta de los impuestos y en el patrimonio se dio un 
Efectos en los estados financieros de la implementación de la gestión ambiental en el molino industrial arrocera superior limitada por Carolina Martínez - Gina Ortiz - José Hernández

incremento positivo por la adsorción de las pérdidas acumuladas de ejercicios anteriores; lo cual indica que la empresa tuvo un crecimiento mínimo, pero que le permite la permanencia en el mercado.

En cuanto al análisis vertical para el año 2014, muestra que la empresa Industria Arrocera Superior Ltda. tiene baja capacidad de respaldo de activos corrientes para cubrir el alto nivel de endeudamiento en pasivos corrientes que posee la organización en cuentas por pagar a menos de un año. Sin embargo, cuenta con un buen respaldo en activos no corrientes que puede respaldar las deudas a corto y largo plazo.

Para la empresa es importante la implementación del sistema de gestión ambiental, porque es una obligación que tiene la empresa el de proteger el medio ambiente, pero conscientes de que esto podría tener afectaciones de tipo económico y legal, por lo que resulta ser una problemática no tener este sistema. En consecuencia y para la implementación del sistema de gestión ambiental en la empresa, y con los referentes teóricos, se avanzó en la propuesta de macro proceso para la implementación de la gestión ambiental en la empresa como se muestra en la figura 1.

En la Fase I - Autoevaluación Inicial: se realiza un diagnóstico inicial sobre las acciones que está realizando la empresa para prevenir las causas y mitigar las consecuencias de los factores que afectan el medio ambiente. En la Fase II Revisión Inicial, se realiza una revisión del proceso de planeación para la ejecución de la gestión ambiental y que actividades se requiere para la implementación de sistema. En la Fase III - Alcance, se identifica de manera precisa la normatividad que debe cumplir la empresa con respecto a la protección del medio ambiente. En la Fase IV - Metodología, se determinan cuáles son los lineamientos metodológicos para la implementación del sistema. En la Fase V Etapas de la Política Ambiental, se realiza una revisión de la política ambiental si existe, en cuanto a los valores, lineamientos y parámetros tratados. En la Fase VI - Desarrollo del Programa del Sistema, se establece el programa del sistema de gestión ambiental dirigido a los objetivos plateados. En la Fase VII - Implementación del sistema, se pone en marcha el programa diseñado y los diferentes lineamientos metodológicos establecidos. En la Fase VIII - Aseguramiento del sistema, se realiza una revisión del sistema y se dispone o presupuesta o aseguran los recursos humanos, físicos y financieros para el desarrollo del sistema. En la Fase IX - Medición y Retroalimentación del Sistema, se realiza las mediciones respectivas del programa, para la verificación de lo establecido con lo realizado.

Este sistema de gestión ambiental debe ser ejecutado por el Departamento de Gestión Ambiental, quien desarrollará todas las fases anteriormente descritas junto con la dirección de la empresa en consonancia con la política de calidad propuesta. Por ello la necesidad de su creación del DGA para lo cual se requiere de seguir los siguientes pasos:

El primer paso es el Diseño y aplicación de un programa de capacitación al personal, en el cual busca establecer las condiciones de conocimiento o competencias del recurso humano actual sobre el tema, con el propósito de diseñar un programa de capacitación que amplié el saber sobre el tema para lograr el diseño e implementación del DGA. Pasó dos: se determina el tamaño del DGA y de su funcionamiento según las exigencias legales. Pasó tres: definición de los recursos para el DGA, se determinan los recursos humanos, técnicos, tecnológicos y físicos que requiere el departamento para su funcionamiento dependiendo del tamaño ya definido; para que la alta gerencia autorice el presupuesto y la ejecución de los recursos monetarios suficientes: Paso cuatro: Montaje del DGA, en el cual con los recursos económicos definidos y presupuestados, se procede a la selección de personal, la adecuación de la estructura física y tecnológica, y todo lo que se requiere para el funcionamiento del departamento. Paso cinco: Diseño de procesos, sistema de gestión ambiental y el proceso de implementación y el paso seis: la puesta en 
Efectos en los estados financieros de la implementación de la gestión ambiental en el molino industrial arrocera superior limitada por Carolina Martínez - Gina Ortiz - José Hernández

marcha del SGA y mejora continua, en el cual se debe estar constantemente realizando auditorias y mejoras al sistemas con el propósito de conseguir los resultados esperados en concordancia con las normas legales exigidas por el Estado.

Al implementar el proceso de gestión ambiental mediante le DGA se logra:

Reducción del pasivo ambiental provocado. Toda acción dentro de la empresa enfocada a evitar desperdicios, a mitigar los efectos negativos de las actividades laborales como al aire, agua, suelo y fauna, lleva a reducir las consecuencias al medio ambiente y hacia la población.

Concientización a la protección del medio ambiente: al incentivar al recurso humano por medio de la capacitación y el reconocimiento a las acciones encaminadas a la protección del medio, lleva a que se cree la suficiente conciencia de apoyar las directrices establecidas por la empresa para evitar las consecuencias del pasivo ambiental que puede provocar la empresa, y mitigar aquellas consecuencias ya provocadas.

Disminución de costos. Al tener mayor eficiencia en los procesos y al reducir el impacto ambiental, se podrá obtener una reducción de los costos, como por ejemplo en el agua, energía, en las actividades productivas y en fin de todo aquello en donde se aplique la tecnología limpia como medio de protección de medio ambiente.

Por lo tanto, al implementarse el DGA se podrá cumplir con la Ley 1124 de 2007 y el Decreto 1299 de 2008, y además cumpliendo con la legislación existente sobre el tema, se evita así pago de multas o sanciones por el incumplimiento.

\section{Conclusiones}

La empresa Industria Arrocera Superior Ltda., no posee un área especializada de gestión ambiental como lo estipula Ley 1124 de 2007 y el Decreto 1299 de 2008, y tiene la necesidad de mejorar la capacitación del recurso humano sobre todo lo referente a la legislación ambiental, la implementación del DGA, importante para mejorar las actividades productivas y para cumplir la normatividad al respecto; porque que, si no, le puede acarrear las sanciones correspondientes. Por lo tanto, no se realiza un proceso de gestión ambiental adecuado a las necesidades existentes mediante la aplicación de unas políticas y actividades encaminadas a mitigar los efectos negativos hacia el medio ambiente, a pesar de que se cuenta con los recursos monetarios para avanzar en la creación del DGA.

Se logró establecer que la empresa Industria Arrocera Superior Ltda., no está cumplido a cabalidad diferentes normas relacionadas con la protección ambiental y también para el desarrollo de la gestión empresarial, que de una u otra manera están afectando o pueden generar consecuencias para la organización y la población circundante. Entre las normas que no han sido cumplidas en su totalidad por la empresa se encuentra el Decreto 1713 de 2002 que se refiere al desarrollo de la Gestión integral de residuos en cuanto a la recolección y almacenamiento de residuos sólidos producidos por la actividad industrial. Otra norma que se está incumpliendo parcialmente es la Ley 1252 de 2008, la cual dicta normas prohibitivas en materia ambiental, referente a los residuos sólidos y desechos peligrosos; porque la esta empresa no tiene procesos enfocados a la minimización de la generación de residuos peligrosos mediante la aplicación de tecnologías ambientalmente limpias.

El análisis al balance general de la empresa muestra que en el año 2014 se dio un aumento en las cuentas del activo por el incremento en las cuentas por cobrar; y en el pasivo en las cuentas por pagar e impuestos; y en el patrimonio se dio un incremento positivo por la adsorción de las pérdidas acumuladas de ejercicios anteriores; lo cual indica que la empresa tuvo un crecimiento mínimo, pero que le permite la estabilidad y permanencia en el mercado. En este año se pudo determinar que existe baja capacidad de respaldo 
Efectos en los estados financieros de la implementación de la gestión ambiental en el molino industrial arrocera superior limitada por Carolina Martínez - Gina Ortiz - José Hernández

de activos corrientes para cubrir el alto nivel de endeudamiento en pasivos corrientes que posee la organización en cuentas por pagar a menos de un año. Sin embargo, cuenta con un buen respaldo en activos no corrientes que puede respaldar las deudas a corto y largo plazo.

Se determinó que, para la implementación del DGA según el Decreto 1299 de 2008 encaminado a dirigir la gestión ambiental; velar por el cumplimiento de la normatividad ambiental; prevenir, minimizar y controlar la generación de cargas contaminantes; se requiere cumplir con los siguientes pasos: determinar el tamaño del DGA y de su funcionamiento según las exigencias legales; definir los recursos para su creación y funcionamiento; realizar el montaje; diseñar los procesos y la forma de poner en marcha el sistema de gestión ambiental y por ultimó se debe mantener un proceso de mejora continua.

\section{Referencias}

Alarcón A, D y Ulloa P, E, I (2012). El análisis de los estados financieros: papel en la toma de decisiones gerenciales. Disponible en: http://www.eumed.net/cursecon/ecolat/cu/2012/a aup.html

Bernal, C. A. (2006). Metodología de la Investigación. Ciudad de México. Segunda edición: Pearson Educación.

Congreso de la República (2007). Ley 1124 de 2007. Por medio de la cual se reglamenta el ejercicio de la profesión de Administrador Ambiental. Bogotá. Congreso de la República.
Constitucional, C. (Julio 22 de 2009). Sentencia 486.

Cordero, M. B. (2012). Gestión ambiental: Camino al desarrollo sostenible. Primera edición San José: Universidad Estatal a Distancia.

Cortes, A. L. (2010). Diagnóstico de la implementación de un sistema de gestión ambiental y sus principales efectos en los estados contables en la empresa editorial leyer. Bogotá: Monografía presentada para optar el título de Licenciado Contadora Pública.

Estupiñán G, R (2006). Análisis Financiero y de Gestión. Segunda edición. Bogotá. Ecoe ediciones Ltda. 2006

Field, B. C., \& Field, M. K. (2003). Economía Ambiental. Madrid. Tercera edición: Mc Graw Hill

Gray, R., \& Bebbington, J. (2001). Contabilidad y Auditoría Ambiental. Segunda edición. Bogotá: Ecoe Ediciones.

Herrera F, A (2010). La obligatoriedad del Departamento de Gestión Ambiental Empresarial y su marco normativo en Colombia. Medellín. Revista Pensamiento Americano

Industria Arrocera Superior Ltda (2015). Información general de la empresa. Acacias. Industria Arrocera Superior Ltda

Vázquez S, M (2009). Desafíos y oportunidades de la gestión ambiental en el ámbito empresarial. Cuba. Universidad de Pinar del Río. 